\title{
Monge-Ampère foliations with singularities at the boundary of strongly convex domains
}

\section{Filippo Bracci • Giorgio Patrizio}

Received: 23 June 2004 / Revised version: 5 January 2005

Published online: 2 April 2005 - @ Springer-Verlag 2005

\begin{abstract}
Let $D \subset \mathbb{C}^{N}$ be a bounded strongly convex domain with smooth boundary. We consider a Monge-Ampère type equation in $D$ with a simple pole at the boundary. Using the Lempert foliation of $D$ in extremal discs, we construct a solution $u$ whose level sets are boundaries of horospheres. Among other things, we show that the biholomorphisms between strongly convex domains are exactly those maps which preserves our solution.
\end{abstract}

\section{Introduction}

Let $D \subset \mathbb{C}^{N}$ be a bounded strongly convex domain with smooth boundary and let $z_{0} \in D$. In his amazing work [15] Lempert constructs a solution $L: D \rightarrow \mathbb{R}$ to the Monge-Ampère equation

$$
\left\{\begin{array}{l}
u \text { plurisubharmonic in } D, \\
(\partial \bar{\partial} u)^{N}(z)=0, \text { for } z \in D \backslash\left\{z_{0}\right\} \\
d u \neq 0 \\
u(z)=0 \text { for } z \in \partial D \\
u(z)-\log \left\|z_{0}-z\right\|=O(1) \text { as } z \rightarrow z_{0}
\end{array}\right.
$$

In fact, Lempert proved that given a bounded strongly convex domain in $\mathbb{C}^{N}$ with smooth boundary, and fixed a point $z_{0} \in D$, for any point $z \in D$ there exists a unique complex geodesic $\varphi: \mathbb{D} \rightarrow D$, i.e., a holomorphic isometry between $\omega$ (the Poincare metric in $\mathbb{D}$ ) and the Kobayashi metric $k_{D}$, with $\varphi(0)=z_{0}$ and $\varphi(t)=z$ for a suitable $t \in(0,1)$ and such that $\varphi$ extends smoothly past the boundary. Furthermore the complex geodesic discs through the point $z_{0}$ provide a foliation of $D$ (singular at $z_{0}$ ) which is exactly the foliation associated to the plurisubharmonic solution $L$ of the complex Monge-Ampère equation (1).

\section{F. BRACCI}

Dipartimento di Matematica, Università di Roma "Tor Vergata", Via della Ricerca Scientifica 1, 00133 Roma, Italy (e-mail: fbracci@mat . uniroma2 . it)

G. PATRIZIO

Dipartimento di Matematica “Ulisse Dini”, Università di Firenze, Viale Morgagni 67-A, 50134 Firenze, Italy (e-mail: patrizio@math.unifi.it) 
It turns out that the solution $L$ is the defining function for the balls centered in $z_{0}$ for the Kobayashi distance. This deep result establishes a surprising tie between intrinsic metrics and potential theory in higher dimension. Lempert's construction is the cornerstone for many impressive constructions in several complex variables.

Later, suitably adapting and pushing further Lempert's arguments, Abate [3] and Chang, Hu and Lee [10] showed that existence and uniqueness for complex geodesic discs hold even if the point $z_{0}$ is chosen at the boundary of the domain $D$ and the point $z$ is allowed to vary in $\bar{D}$. In this case they show that there exists a complex geodesic $\varphi: \mathbb{D} \rightarrow D$ which extends smoothly to the boundary and with $z_{0}=\varphi(1)$ and $z$ in $\overline{\varphi(\mathbb{D})}$. The map $\varphi$ is unique up to composition of automorphisms of the unit disc and the parametrization may be chosen uniquely fixing suitable extremal conditions at the point $z_{0}$. It is natural to ask whether it is possible to interpret also in this case the foliation of complex geodesics passing through the boundary point $z_{0}$ as the foliation associated to a solution of the complex homogeneous Monge-Ampère equation. The main result of this work is to show that indeed this is the case.

Heuristically, as in the unit disc the Green potential is replaced by the Poisson kernel when the pole goes to the boundary and the type of singularity changes from a logarithmic to a simple singularity, even in our case, as $D \ni z_{0} \rightarrow p \in \partial D$, one can expect to replace the logarithmic singularity with a simple pole. Thus we introduce and study the following Monge-Ampère equation with a singularity at a boundary point $p \in \partial D$ :

$$
\left\{\begin{array}{l}
u \text { plurisubharmonic in } D \\
(\partial \bar{\partial} u)^{N} \equiv 0 \\
d u \neq 0 \\
u(z)=0 \text { for } z \in \partial D \backslash\{p\} \\
u(z) \approx\|p-z\|^{-1} \text { as } z \rightarrow p \text { non-tangentially }
\end{array}\right.
$$

Where, for real or complex functions $a(z), b(z)$, the symbol $a(z) \approx b(z)$ for $z \rightarrow p$ means that there exist $c, C>0$ such that $c|b(z)| \leq|a(z)| \leq C|b(z)|$ for all $z$ close enough to $p$.

Using the work of Chang, Hu and Lee [10], in Theorem 6.3, we show that (2) has a smooth solution on $D$ such that $(\partial \bar{\partial} u)^{N-1} \neq 0$. Our solution $u$ is "natural" in the sense that its level sets are exactly the boundaries of the horospheres of $D$ at $p$. Horospheres are "limits of Kobayashi balls", defined by Abate by means of the Kobayashi distance or by means of Busemann functions (see, e.g., [1], [2] and section 3) and they are one of the main tools in the study of iteration theory.

Indeed, the construction of our solution to (2) is very much related to the understanding of geometrical properties of horospheres, which we study in details in section 3. Bland, Duchamp and Kalka in [6] (see also [18]) proved that a biholomorphism between two strongly convex domains is characterized by the property 
of being a biholomorphism between any two Kobayashi balls of the same radius. As a spin off result, quite interesting by its own, we show that the same property holds for horospheres (see section 4):

Theorem 1.1. Let $D, D^{\prime}$ be bounded strongly convex domains in $\mathbb{C}^{N}$ with smooth boundary. Then $D$ is biholomorphic to $D^{\prime}$ if and only if there exist a horosphere $E_{D} \subset D$ with center $p \in \partial D$, a horosphere $E_{D^{\prime}} \subset D^{\prime}$ with center $q \in \partial D^{\prime}$ and a biholomorphism $F: E_{D} \rightarrow E_{D^{\prime}}$ such that $F(p)=q$ (in the sense of non-tangential limits) and the radius of $E_{D}$ with respect to some $z \in E_{D}$ is equal to the radius of $E_{D^{\prime}}$ with respect to $F(z)$.

For the solution of the Monge-Ampère equation with logarithmic singularity at an internal point, the associated Monge-Ampère foliation is a singular foliation (holomorphic if and only if the domain is biholomorphic to a complete circular domain, the Kobayashi indicatrix at that point, see [19], [20]). If the foliation has singularity on the boundary we show that the associated Monge-Ampère foliation is actually a smooth fibration with base $\mathbb{C}^{N-1}$ and fiber the unit disc $\mathbb{D}$ (see Theorem 3.5).

Finally, we prove the following boundary Schwarz-type result:

Theorem 1.2. Let $D, D^{\prime} \subset \mathbb{C}^{N}$ be bounded strongly convex domains with smooth boundary. Let $p \in \partial D$ and $q \in \partial D^{\prime}$. Let $u_{D}$ (respectively $u_{D^{\prime}}$ ) be the solution of (2) in $D$ (respectively in $D^{\prime}$ ). Let $F: D \rightarrow D^{\prime}$ be holomorphic and assume that $F$ is continuous at $p$. Then $F$ is a biholomorphism such that $F(p)=q$ if and only if there exists $\lambda \in \mathbb{R}^{+} \backslash\{0\}$ such that $F^{*}\left(u_{D^{\prime}}\right)=\lambda u_{D}$.

The plan of the paper is as follows. In Section 2 we recall some preliminary classical results in the unit disc, as needed for our aim. In Section 3 we discuss the results of Chang-Hu-Lee in terms of "Monge-Ampère foliations" showing that actually the foliation in complex geodesics centered at $p \in \partial D$ is a fibration. In Section 4 we introduce horospheres and prove some technical lemmas about them. In Section 5 we discuss mappings of horospheres onto horospheres and prove Theorem 1.1. In Section 6 we construct the solution of (2). Finally, in Section 7 we relate our work with Busemann functions and prove Theorem 1.2.

We conclude this introduction remarking that the smoothness required for the boundary of $\partial D$ can be lowered up to $C^{k}, k \geq 14$ as in [10] (see also [14] where it is shown that actually $C^{3}$ is enough for much of the construction). Also, instead of working with strongly convex domains one could work with strictly linearly convex domains.

\section{Preliminary on the Julia-Wolff-Carathéodory theorems}

In the sequel we will use several times the classical Julia Lemma, Wolff Lemma and Julia-Wolff-Carathéodory Theorem. For the reader convenience we state here 
such theorems (in the form we need) and refer to the book [1] for proofs. Also, we state and prove a corollary of the Julia-Wolff-Carathéodory Theorem we will need later.

As a matter of notation, if $D$ is a domain in $\mathbb{C}^{n}$ and $\left\{z_{k}\right\} \subset D$ is a sequence which converges to $p \in \partial D$, we say that $z_{k} \rightarrow p$ non-tangentially if there exists a constant $c>0$ such that, for $k \rightarrow \infty$,

$$
\left\|z_{k}-p\right\| \leq c \cdot \operatorname{dist}\left(z_{k}, \partial D\right) .
$$

Let $P(\zeta)=\left(1-|\zeta|^{2}\right)\left(|1-\zeta|^{2}\right)^{-1}$ be the Poisson kernel in the unit disc $\mathbb{D}:=\{\zeta \in \mathbb{C}:|\zeta|<1\}$. The horosphere of center 1 , pole 0 and radius $R>0$ is given by $E_{\mathbb{D}}(1,0, R):=\{\zeta \in \mathbb{D}: P(\zeta)>1 / R\}$ (we refer to Heins [12] for explanations and developments of the relations between horospheres and Poisson kernel).

The first result we recall is a simple consequence of Julia's Lemma:

Lemma 2.1. Let $f: \mathbb{D} \rightarrow \mathbb{D}$ be holomorphic. Suppose there exists $\alpha \in(0,+\infty)$ such that for all $R>0$

$$
f\left(E_{\mathbb{D}}(1,0, R)\right) \subset E_{\mathbb{D}}(1,0, \alpha R),
$$

and suppose there exists $R_{0}>0$ and $\zeta \in \partial E_{\mathbb{D}}\left(1,0, R_{0}\right)$ such that $f(\zeta) \in$ $\partial E_{\mathbb{D}}\left(1,0, \alpha R_{0}\right)$. Then $f$ is an automorphism of $\mathbb{D}$.

Conversely, if $f$ is an automorphism of $\mathbb{D}$ such that $f(1)=1$, then there exists $\alpha \in(0,+\infty)$ such that $f\left(E_{\mathbb{D}}(1,0, R)\right)=E_{\mathbb{D}}(1,0, \alpha R)$ for all $R>0$.

Let $\omega$ denote the Poincaré distance on $\mathbb{D}$. By the very definition $\omega\left(\zeta_{1}, \zeta_{2}\right)=$ $\frac{1}{2} \log \frac{1+\left|T_{\zeta_{1}}\left(\zeta_{2}\right)\right|}{1-\left|T_{\zeta_{1}}\left(\zeta_{2}\right)\right|}$ where $T_{\zeta_{1}}$ is any automorphism of $\mathbb{D}$ mapping $\zeta_{1}$ to 0 . For $f$ : $\mathbb{D} \rightarrow \mathbb{D}$ holomorphic such that $f(1)=1$ (in the sense of non-tangential limits) we let

$$
\frac{1}{2} \log f^{\prime}(1):=\liminf _{\zeta \rightarrow 1}[\omega(0, \zeta)-\omega(0, f(\zeta))]=\liminf _{\zeta \rightarrow 1} \frac{1}{2} \log \frac{1-|f(\zeta)|}{1-|\zeta|}
$$

Then we have

Theorem 2.2 (Julia-Wolff-Carathéodory). Let $f: \mathbb{D} \rightarrow \mathbb{D}$ be holomorphic and such that $f(1)=1$ (in the sense of non-tangential limits). Assume that $f^{\prime}(1)<\infty$. Then

(1) $\lim _{\mathbb{R} \ni r \rightarrow 1^{-}}[\omega(0, r)-\omega(0, f(r))]=\lim _{\mathbb{R} \ni r \rightarrow 1^{-}} \frac{1}{2} \log \frac{1-|f(r)|}{1-r}=\frac{1}{2} \log f^{\prime}(1)$.

(2) The function $\zeta \mapsto \frac{1-f(\zeta)}{1-\zeta}$ has limit $f^{\prime}(1)$ for $\zeta \rightarrow 1$ non-tangentially.

(3) The function $f^{\prime}(\zeta)$ has limit $f^{\prime}(1)$ for $\zeta \rightarrow 1$ non-tangentially.

Also we have the following boundary Schwarz-type lemma, due in this form to Herzig [13] (see also [10, Lemma 2]): 
Theorem 2.3 (Herzig). Let $f: \mathbb{D} \rightarrow \mathbb{D}$ be holomorphic and such that $f(1)=1$ (in the sense of non-tangential limits). If $f(0)=0$ then $f^{\prime}(1)>1$ unless $f(\zeta)=\zeta$ for all $\zeta \in \mathbb{D}$.

Now we state and prove a corollary which will be used later.

Lemma 2.4. Let $f: E_{\mathbb{D}}(1,0, R) \rightarrow \mathbb{D}$ be holomorphic and let $R>0$. Suppose $\lim _{\mathbb{R} \ni r \rightarrow 1} f(r)=1$ and

$$
\lim _{\mathbb{R} \ni r \rightarrow 1^{-}} \frac{1-|f(r)|}{1-r}=\alpha<\infty .
$$

Then for any sequence $\left\{\zeta_{n}\right\} \subset E_{\mathbb{D}}(1,0, R)$ which converges radially to 1 , i.e., such that $\left|1-\zeta_{n}\right| /\left(1-\left|\zeta_{n}\right|\right) \rightarrow 1$ as $n \rightarrow \infty$, it follows

$$
\lim _{n \rightarrow \infty} \frac{1-\left|f\left(\zeta_{n}\right)\right|}{1-\left|\zeta_{n}\right|}=\alpha
$$

Proof. Let $R>0$ and $g:=f \circ \theta_{R}: \mathbb{D} \rightarrow \mathbb{D}$, where $\theta_{R}$ is defined by

$$
\theta_{R}(\zeta):=\frac{1+R \zeta}{1+R}
$$

Note that $\theta_{R}(r) \in \mathbb{R}$ if $r \in[0,1)$. Then

$$
\lim _{\mathbb{R} \ni r \rightarrow 1^{-}} \frac{1-|g(r)|}{1-r}=\lim _{\mathbb{R} \ni r \rightarrow 1^{-}} \frac{1-\left|f\left(\theta_{R}(r)\right)\right|}{1-\theta_{R}(r)} \cdot \frac{1-\theta_{R}(r)}{1-r}=\alpha \cdot \frac{R}{R+1} .
$$

Namely, by the Julia-Wolff-Carathéodory Theorem 2.2.(1),

$$
\lim _{\mathbb{R} \ni r \rightarrow 1^{-}}[\omega(0, r)-\omega(0, g(r))]=\frac{1}{2} \log \frac{\alpha R}{R+1} .
$$

Let $t_{n}:=\theta_{R}^{-1}\left(\zeta_{n}\right)$. Then for all $r \in(0,1)$ it follows

$$
\begin{aligned}
\omega\left(0, t_{n}\right)-\omega\left(0, g\left(t_{n}\right)\right)= & \omega\left(0, t_{n}\right)+\omega\left(r, t_{n}\right)-\omega(0, r) \\
& +\omega(0, r)-\omega\left(r, t_{n}\right)-\omega\left(0, g\left(t_{n}\right)\right) \\
\leq & \omega\left(0, t_{n}\right)+\omega\left(r, t_{n}\right)-\omega(0, r) \\
& +\omega(0, r)-\omega\left(g(r), g\left(t_{n}\right)\right)-\omega\left(0, g\left(t_{n}\right)\right) \\
\leq & {\left[\omega\left(0, t_{n}\right)+\omega\left(r, t_{n}\right)-\omega(0, r)\right] } \\
& +[\omega(0, r)-\omega(0, g(r))],
\end{aligned}
$$

where we used that $\omega\left(g(r), g\left(t_{n}\right)\right) \leq \omega\left(r, t_{n}\right)$ and the triangle inequality. Taking into account that for all $z \in \mathbb{D}$

$$
\lim _{w \rightarrow 1}[\omega(z, w)-\omega(0, w)]+\omega(0, z)=\frac{1}{2} \log \frac{|1-z|}{1-|z|},
$$


if we let $r \rightarrow 1$ in (5), by (4) we get

$$
\omega\left(0, t_{n}\right)-\omega\left(0, g\left(t_{n}\right)\right) \leq \frac{1}{2} \log \frac{\left|1-t_{n}\right|}{1-\left|t_{n}\right|}+\frac{1}{2} \log \frac{\alpha R}{R+1} .
$$

By hypothesis $\zeta_{n} \rightarrow 1$ radially. Since $\left(\theta_{R}^{-1}\right)^{\prime}(1) \in \mathbb{R}$ it follows that $t_{n}$ converges to 1 radially as well. Therefore the right-hand side of (6) tends to $\frac{1}{2} \log \frac{\alpha R}{R+1}$ as $n \rightarrow \infty$. Recalling Theorem 2.2.(1) and (4), we see that the left-hand side of (6) tends to the same limit as $n \rightarrow \infty$. Furthermore,

$$
\begin{aligned}
\lim _{n \rightarrow \infty}\left[\omega\left(0, t_{n}\right)-\omega\left(0, g\left(t_{n}\right)\right)\right] & =\frac{1}{2} \log \lim _{n \rightarrow \infty} \frac{1-\left|f\left(\zeta_{n}\right)\right|}{1-\left|\zeta_{n}\right|} \frac{1-\left|\zeta_{n}\right|}{1-\left|\theta_{R}^{-1}\left(\zeta_{n}\right)\right|} \\
& =\frac{1}{2} \log \left[\frac{R}{R+1} \lim _{n \rightarrow \infty} \frac{1-\left|f\left(\zeta_{n}\right)\right|}{1-\left|\zeta_{n}\right|}\right],
\end{aligned}
$$

from which the statement follows.

\section{Monge-Ampère foliation at the boundary}

Let $D$ be a bounded strongly convex domain in $\mathbb{C}^{N}$ with smooth boundary. By Lempert's work (see [15] and [1]), adapted by Abate (see [3]) and Chang, Hu and Lee (see [10]) given any point $z \in \bar{D}$ there exists a unique complex geodesic $\varphi: \mathbb{D} \rightarrow$ D, i.e., a holomorphic isometry between $\omega$ (the Poincaré metric in D) and $k_{D}$ (the Kobayashi distance in $D$ ), such that $\varphi$ extends smoothly past the boundary, $\varphi(0)=z_{0}$ and $\varphi(t)=z$, with $t \in(0,1)$ if $z \in D$ and $t=1$ if $z \in \partial D$. Moreover for any such complex geodesic there exists a holomorphic retraction $\rho: D \rightarrow \varphi(\mathbb{D})$, i.e., $\rho$ is a holomorphic self-map of $D$ such that $\rho \circ \rho=\rho$ and $\rho(z)=z$ for any $z \in \varphi(\mathbb{D})$. We call such a $\rho$ the Lempert projection associated to $\varphi$. Furthermore we let $\tilde{\rho}:=\varphi^{-1} \circ \rho$ and call it the left inverse of $\varphi$, for $\tilde{\rho} \circ \varphi=I d_{\mathbb{D}}$. The triple $(\varphi, \rho, \tilde{\rho})$ is the so-called Lempert projection device.

As we remarked for instance in $[8, \mathrm{p} .145]$ the maps $\varphi, \tilde{\rho}$ are unique only up to "parametrization" (i.e., if $\theta \in \operatorname{Aut}(\mathbb{D})$ then $\varphi \circ \theta$ is a complex geodesic and $\theta^{-1} \circ \varphi$ is the associated left-inverse) while $\rho$ is unique (that is, depends only on the image $\varphi(\mathbb{D}))$.

Remark 3.1. Assume $\varphi: \mathbb{D} \rightarrow D$ is a complex geodesic and let $\tilde{\rho}: D \rightarrow \mathbb{D}$ be its left inverse. By [17, Proposition 1 p. 345] it follows that $\tilde{\rho}(\bar{D} \backslash \varphi(\partial \mathbb{D})) \subset \mathbb{D}$. In particular, if $\eta: \mathbb{D} \rightarrow D$ is a complex geodesic such that $\tilde{\rho}(\eta(\mathbb{D}))=\mathbb{D}$ or, equivalently, $\rho(\eta(\mathbb{D}))=\varphi(\mathbb{D})$ (where $\rho: D \rightarrow D$ is the Lempert projection associated to $\varphi$ ) then $\eta(\mathbb{D})=\varphi(\mathbb{D})$.

With an abuse of notation, we call "complex geodesic" also the image of a complex geodesic $\varphi: \mathbb{D} \rightarrow D$. We let $\mathcal{F}_{x}$ denote the foliation of $D$ defined by all the complex geodesics whose closure contain $x \in \partial D$. Thus an element $G \in \mathcal{F}_{x}$ 
is just a one-dimensional holomorphic retract of $D$. We call $\mathcal{F}_{x}$ a (boundary) Monge-Ampère foliation.

Proposition 3.2. Let $D$ be a bounded strongly convex domain in $\mathbb{C}^{N}$ with smooth boundary. Let $x \in \partial D$. Then $\mathcal{F}_{x}$ is a smooth foliation of $D$ whose leaves are complex geodesics of $D$.

Proof. Let $z \in D$. We are going to show that there exists a open subset $V_{z} \subset D$ containing $z$ and a smooth map $h: V_{z} \mapsto U_{z} \times \mathbb{D} \subset \mathbb{C}^{N-1} \times \mathbb{D}$ which trivializes $\mathcal{F}_{x} \cap V_{z}$; namely, for all $w \in U_{z}$ the map $\mathbb{D} \ni \zeta \mapsto h^{-1}(w, \zeta)$ is a leaf of $\mathcal{F}_{x}$. This is essentially the content of [10, Theorem 3]. However, for the reader convenience, we give here a proof using a different choice of coordinates. We proceed this way. Let $G$ be the complex geodesic containing $z$ and let $\rho_{z}: D \rightarrow G$ be the associated Lempert's projection. By [15, Proposition 9 ] (see also [17, Proposition 11]) we can assume that $G=\mathbb{D} \times\{(0, \ldots, 0)\}$, that $z=(0, \ldots, 0)$ and that $\rho_{G}\left(\left(z_{1}, \ldots, z_{N}\right)\right)=\left(z_{1}, 0, \ldots, 0\right)$. Let $\tilde{V}_{z}$ be a small ball centered at $z=(0, \ldots, 0)$. We let $U_{z}^{\prime}:=\tilde{V}_{z} \cap \rho_{G}^{-1}(z)$. Then $U_{z}^{\prime}=\{0\} \times U_{z}$ with $U_{z}$ an open ball in $\mathbb{C}^{N-1}$ centered at 0 . It is clear that if $\tilde{V}_{z}$ is small enough then $U_{z}^{\prime}$ is an $N-1$ complex affine manifold transverse to $\mathcal{F}_{x}$. Now let $\mathcal{A}_{z} \subset \mathcal{F}_{x}$ be the set of one dimensional holomorphic retracts which intersect $U_{z}^{\prime}$. We set

$$
V_{z}:=\bigcup_{G \in \mathcal{A}_{z}} G
$$

The set $V_{z}$ is open in $D$. This follows at once from the fact that the space of (the closure of) one dimensional holomorphic retracts of $D$ with, for instance, the topology of uniform convergence on compacta is homeomorphic to the space of (the closure of) complex geodesic sets endowed with the Hausdorff topology of compacta of $\bar{D}$ (see [8, Lemma 5.3]). However this will also follow a fortiori from the fact that $V_{z}$ is the homeomorphic image of $U_{z} \times \mathbb{D}$.

Now we define $h: V_{z} \rightarrow U_{z} \times \mathbb{D}$ as follows. For any $w \in U_{z}^{\prime}$ we let $\varphi_{w}: \mathbb{D} \rightarrow$ $D$ be the unique complex geodesic such that $\varphi_{w}(0)=w$ and $\varphi_{w}(1)=x$. Thus, if $u \in V_{z}$ then $u=\varphi_{w}(\zeta)$ for a unique $w \in U_{z}^{\prime}$ and a unique $\zeta \in \mathbb{D}$. Therefore we set

$$
V_{z} \ni u \mapsto h(u):=(w, \zeta) \in U_{z} \times \mathbb{D},
$$

where, with some abuse of notation we call $w$ both the element of $U_{z}^{\prime}$ and its projection to $U_{z}$. We claim that $h$ is a smooth diffeomorphism with inverse

$$
U_{z} \times \mathbb{D} \mapsto h^{-1}(w, \zeta):=\varphi_{w}(\zeta) \in V_{z} .
$$

Since any complex geodesic parameterizing $\varphi_{w}(\mathbb{D})$ is obtained by pre-composing with automorphisms of $\mathbb{D}$ then we can use [10, Proposition 5'] (see also the argument at $\left[10\right.$, p. 369] and [15, pp. 460-461]) to show that $h, h^{-1}$ are smooth. 
From the proof of Proposition 3.2 it follows that the local trivializing coordinates $(w, \zeta) \in U_{z} \times \mathbb{D}$ introduced are holomorphic in $\zeta$, and thus they are adapted in the sense of [6]. Thus, using [6, Lemma 3.2] and arguing as at [6, p. 27] we have the following lemma which will be useful later:

Lemma 3.3. Let $D, D^{\prime}$ be bounded strongly convex domains with smooth boundary in $\mathbb{C}^{N}$. Let $p \in \partial D$ and let $U$ be an open subset of $D$ intersecting each leaf of the boundary Monge-Ampère foliation $\mathcal{F}_{p}$. Let $\tilde{F}: D \rightarrow D^{\prime}$ be a smooth $\left(C^{1}\right.$ is enough) map such that

(1) $\tilde{F}$ is holomorphic on $U$,

(2) for any $\varphi: \mathbb{D} \rightarrow D$ complex geodesic such that $\varphi(\mathbb{D}) \in \mathcal{F}_{p}$ the map $\mathbb{D} \ni \zeta \mapsto F(\varphi(\zeta)) \in D^{\prime}$ is holomorphic.

Then $\tilde{F}$ is holomorphic on $D$.

Using the boundary spherical representation of Chang, Hu and Lee (see [10, Theorem 3]) one can refine Proposition 3.2 in order to obtain "global coordinates" adapted to the boundary Monge-Ampère foliation. For the reader convenience and since it will be useful later, we recall here the Chang, $\mathrm{Hu}$ and Lee construction as needed for our aim. Let $p \in \partial D$ and, up to rigid transformation, assume that the unit normal vector for $\partial D$ at $p$ is $e_{1}=(1, \ldots, 0)$. Let $L_{p}:=\left\{v=\left(v_{1}, \ldots, v_{n}\right) \in \mathbb{C}^{N} \mid\|v\|=1, v_{1}>0\right\}$. For any $v \in L_{p}$ the map $\eta_{v}: \mathbb{D} \ni \zeta \mapsto e_{1}+(\zeta-1) v_{1} v$ is a complex geodesic of $\mathbb{B}^{N}, \eta_{v}(1)=e_{1}$ and $\eta_{v}^{\prime}(1)=v_{1} v$. In [10] Chang, Hu and Lee prove that one can perform a unique choice of a complex geodesic $\varphi_{v}: \mathbb{D} \rightarrow D$ such that $\varphi(1)=p$ and $\varphi^{\prime}(1)=v_{1} v$ imposing an extremality condition on the second derivative of $\varphi$ at 1 (we do not state here such a condition since we do not need it). Then the map $\Phi: D \rightarrow \mathbb{B}^{N}$ is defined as follows:

$$
\Phi(z)=e_{1}+\left(\zeta_{z}-1\right) v_{1} v,
$$

where $\zeta_{z} \in \mathbb{D}$ and $v \in L_{p}$ are the unique data such that $\varphi_{v}\left(\zeta_{z}\right)=z$. The map $\Phi$ is a smooth diffeomorphism whose inverse is easily seen to be

$$
\Phi^{-1}(w)=\varphi_{v}\left(\zeta_{w}\right),
$$

where $\zeta_{w} \in \mathbb{D}$ and $v \in L_{p}$ are the unique data such that $w=\eta_{v}\left(\zeta_{w}\right)$. Moreover $\Phi, \Phi^{-1}$ extend continuously up to the boundary. For future reference, we note here the following fact:

Remark 3.4. For any $v \in L_{p}$ it follows that $\Phi \circ \varphi_{v}=\eta_{v}$. Now, let $\varphi: \mathbb{D} \rightarrow D$ be a complex geodesic such that $\varphi(1)=p$. Then there exists $v \in L_{p}$ and an automorphism $\theta: \mathbb{D} \rightarrow \mathbb{D}$ with $\theta(1)=1$ such that $\varphi=\varphi_{v} \circ \theta$. Notice that $\theta^{\prime}(1)>0$. Therefore, denoting by $\langle\cdot, \cdot\rangle$ the standard Hermitian product in $\mathbb{C}^{N}$,

$$
\begin{aligned}
\left\langle\varphi^{\prime}(1), e_{1}\right\rangle & =\theta^{\prime}(1)\left\langle\varphi_{v}(1), e_{1}\right\rangle=v_{1}^{2} \theta^{\prime}(1)=\theta^{\prime}(1)\left\langle\left(\Phi \circ \varphi_{v}\right)^{\prime}(1), e_{1}\right\rangle \\
& =\left\langle(\Phi \circ \varphi)^{\prime}(1), e_{1}\right\rangle .
\end{aligned}
$$


In particular it follows that $\Phi$ is holomorphic on the leaves of the boundary Monge-Ampère foliation $\mathcal{F}_{p}$ and sends leaves of $\mathcal{F}_{p}$ onto leaves of $\mathcal{G}_{e_{1}}$, the Monge-Ampère foliation of $\mathbb{B}^{N}$ at $e_{1}$.

Using the spherical representation of Chang, $\mathrm{Hu}$ and Lee we can prove the following result:

Theorem 3.5. Let $D$ be a bounded strongly convex domain in $\mathbb{C}^{N}$ with smooth boundary. Let $x \in \partial D$. Then $\mathcal{F}_{x}$ is a smooth fibration of $D$ onto $\mathbb{C}^{N-1}$ with fiber $\mathbb{D}$.

Proof. Since the boundary spherical representation $\Phi: D \rightarrow \mathbb{B}^{N}$ sends the boundary Monge-Ampère foliation $\mathcal{F}_{x}$ to the boundary Monge-Ampère foliation $\mathcal{G}_{e_{1}}$ of the ball $\mathbb{B}^{N}$ (here $e_{1}=(1,0, \ldots, 0)$ ), it is enough to show that the foliation $\mathcal{G}_{e_{1}}$ is a fibration of $\mathbb{B}^{N}$. Write the ball as the upper Siegel plane $\mathbb{H}^{N}=\left\{(z, w) \in \mathbb{C} \times \mathbb{C}^{N-1}: \operatorname{Im} z>\|w\|^{2}\right\}$ using a biholomorphic transformation which sends $e_{1}$ to $\infty$. We claim that the boundary Monge-Ampère foliation of $\mathbb{H}^{N}$ at $\infty$ is given by $\mathcal{G}_{\infty}=\{w=$ const $\}$. Indeed, $\{w=0\}$ is clearly a complex geodesic. The others can be found by translating this by means of parabolic automorphisms of $\mathbb{H}^{N}$ fixing $\infty$, giving the claim. Then $\mathcal{G}_{\infty}$ is clearly a (holomorphic) fibration on $\mathbb{C}^{N-1}$ with fiber (biholomorphic to) $\mathbb{D}$.

In the previous proof we showed that the boundary Monge-Ampère foliation $\mathcal{G}_{e_{1}}$ is actually holomorphic in $\mathbb{B}^{N}$. Also, note that the fibration $\mathcal{G}_{e_{1}}$ is smoothly equivalent to the product $\mathbb{C}^{N-1} \times \mathbb{D}$ but it is clearly not holomorphically equivalent to it. It would be interesting to characterize those domains for which the boundary Monge-Ampère foliation is a holomorphic fibration.

\section{Horospheres}

Let $D$ be a strongly convex bounded domain in $\mathbb{C}^{N}$ with smooth boundary. Let $z_{0} \in D$. We denote by $k_{D}$ the Kobayashi distance in $D$. Following Abate [1], [2] we give the following

Defnition 4.1. A horosphere $E_{D}\left(x, z_{0}, R\right)$ of center $x \in \partial D$, pole $z_{0}$ and radius $R>0$ is defined as

$$
E_{D}\left(x, z_{0}, R\right):=\left\{z \in D: \lim _{w \rightarrow x}\left[k_{D}(z, w)-k_{D}\left(z_{0}, w\right)\right]<\frac{1}{2} \log R\right\} .
$$

It is a feature of strongly convex domain (see, e.g., [1, Theorem 2.6.47]) that the limit defining $E_{D}\left(x, z_{0}, R\right)$ actually does exist.

Remark 4.2. It is known after Abate (see, Corollary 2.6.49 in [1] and [2]) that the horospheres of center $x \in \partial D$ are convex subsets of $D$, smooth and strongly convex at $x$. 
If $D=\mathbb{D}$ the unit disc in $\mathbb{C}$ then

$$
E_{\mathbb{D}}(1,0, R)=\left\{\zeta \in \mathbb{D}: \frac{|1-\zeta|^{2}}{1-|\zeta|^{2}}<R\right\}
$$

is a disc of radius $R /(R+1)$ tangent to $\mathbb{D}$ at 1 .

Remark 4.3. It should be noted that there is another (equivalent) way of defining horospheres, namely by means of the Busemann functions as follows (see [9], [5] and [21]). Let $\varphi: \mathbb{D} \rightarrow D$ be a complex geodesic, $\varphi(1)=p \in \partial D$. Let $s$ be the arc length parameter of the real geodesic $\mathbb{R}^{+} \ni t \mapsto \varphi(t)$ (thus $s(t)=$ $\left.\frac{1}{2} \log \frac{1+t}{1-t}=\omega(0, t)\right)$. Let $z \in D$. The function $k_{D}(z, \varphi(s))-s$ is decreasing in $s$ and, by the triangle inequality, it is bounded by $k_{D}(z, \varphi(0))$. Thus the limit for $s \rightarrow \infty$ does exist. We let

$$
\begin{aligned}
B_{\varphi}(z) & :=\lim _{s \rightarrow+\infty}\left[k_{D}(z, \varphi(s))-s\right]=\lim _{t \rightarrow 1}\left[k_{D}(z, \varphi(t))-k_{D}(\varphi(0), \varphi(t))\right] \\
& =\lim _{t \rightarrow 1}\left[k_{D}(z, \varphi(t))-\omega(0, t)\right],
\end{aligned}
$$

the Busemann function of $\varphi$ at $p$. By construction it follows at once that the set $\left\{z \in D: B_{\varphi}(z)<\frac{1}{2} \log R\right\}$ is the horosphere of $D$ centered at $\varphi(1)=p$ with pole $\varphi(0)$ and radius $R>0$.

Lemma 4.4. Let $G \in \mathcal{F}_{x}$. Then for all $R>0$ it follows that $G \cap E_{D}\left(x, z_{0}, R\right)$ is a complex geodesic of $E_{D}\left(x, z_{0}, R\right)$.

Proof. Fix $R>0$. Let $\rho_{G}: D \rightarrow G$ be the Lempert projection associated to $G$. Then we claim that

$$
\rho_{G}\left(E_{D}\left(x, z_{0}, R\right)\right)=E_{D}\left(x, z_{0}, R\right) \cap G .
$$

Indeed, let $\varphi_{G}: \mathbb{D} \rightarrow D$ be a parametrization for $G$ such that $\varphi_{G}(1)=x$. Since $\rho_{G} \circ \varphi_{G}=\varphi_{G}$, if $z \in E_{D}\left(x, z_{0}, R\right)$ we have

$$
\begin{aligned}
& \lim _{w \rightarrow x}\left[k_{D}\left(\rho_{G}(z), w\right)-k_{D}\left(z_{0}, w\right)\right]=\lim _{r \rightarrow 1}\left[k_{D}\left(\rho_{G}(z), \rho_{G}\left(\varphi_{G}(r)\right)\right)-k_{D}\left(z_{0}, \varphi_{G}(r)\right)\right] \\
& \quad \leq \lim _{r \rightarrow 1}\left[k_{D}\left(z, \varphi_{G}(r)\right)-k_{D}\left(z_{0}, \varphi_{G}(r)\right)\right]<\frac{1}{2} \log R .
\end{aligned}
$$

Therefore $\rho_{G}(z) \in E_{D}\left(x, z_{0}, R\right) \cap G$. This means that $E_{D}\left(x, z_{0}, R\right) \cap G$ is a (one-dimensional) holomorphic retract of $E_{D}\left(x, z_{0}, R\right)$ whose closure contains $x$. By [22, Théorème 2.3] it follows that $E_{D}\left(x, z_{0}, R\right) \cap G$ is actually a complex geodesic.

Remark 4.5. Let $E_{D}\left(p, z_{0}, R\right)$ be a horosphere in $D$. Let $G$ be a complex geodesic for $D$ such that $z_{0} \in G$ and let $\varphi_{G}: \mathbb{D} \rightarrow D$ be a parametrization such that $\varphi_{G}(1)=p, \varphi_{G}(0)=z_{0}$. Letting $w=\varphi_{G}(r)$ for $r \rightarrow 1$ in (7) one easily sees that $\varphi_{G}\left(E_{\mathbb{D}}(1,0, R)\right)=E_{D}\left(p, z_{0}, R\right) \cap G$. Let $\theta_{R}$ be as in (3). Then $\theta_{R}(\mathbb{D})=E_{\mathbb{D}}(1,0, R)$ and $\varphi \circ \theta_{R}: \mathbb{D} \rightarrow G \cap E_{D}\left(p, z_{0}, R\right)$ is a parametrization of the complex geodesic $G \cap E_{D}\left(p, z_{0}, R\right)$ of $E_{D}\left(p, z_{0}, R\right)$. 
Now we see how the radius of the horosphere changes when changing the pole. Let $p \in \partial D$ and let $z_{1} \in D$. Set

$$
\frac{1}{2} \log r\left(z_{1}\right):=\lim _{w \rightarrow p}\left[k_{D}\left(z_{0}, w\right)-k_{D}\left(z_{1}, w\right)\right] .
$$

Then

$$
E_{D}\left(p, z_{0}, R\right)=E_{D}\left(p, z_{1}, R \cdot r\left(z_{1}\right)\right) .
$$

Indeed,

$$
k_{D}(z, w)-k_{D}\left(z_{1}, w\right)=\left[k_{D}(z, w)-k_{D}\left(z_{0}, w\right)\right]+\left[k_{D}\left(z_{0}, w\right)-k_{D}\left(z_{1}, w\right)\right],
$$

and since the limit for $w \rightarrow p$ exists then we have (11).

In particular for any complex geodesic $\varphi: \mathbb{D} \rightarrow D$ such that $\varphi(1)=p$ it follows that

$$
\varphi\left(E_{\mathbb{D}}(1,0, R \cdot r(\varphi(0)))\right)=\varphi(\mathbb{D}) \cap E_{D}\left(p, z_{0}, R\right) .
$$

As a final result of this section, we obtain a technical lemma which will be useful in the sequel.

Lemma 4.6. Let $E_{D}:=E_{D}\left(p, z_{0}, R\right)$ be a horosphere in $D$ with $z_{0} \in E_{D}$, i.e., $R>1$. Let $\varphi: \mathbb{D} \rightarrow$ D be a complex geodesic such that $\varphi(1)=p$ and $\varphi(0) \in E_{D}$. Then

$$
\lim _{\mathbb{R} \ni r \rightarrow 1^{-}}\left[k_{D}\left(z_{0}, \varphi(r)\right)-k_{E_{D}}\left(z_{0}, \varphi(r)\right)\right]=\frac{1}{2} \log \frac{R-1}{R} .
$$

Proof. Let $\varphi_{o}: \mathbb{D} \rightarrow D$ be the complex geodesic such that $\varphi_{o}(0)=z_{0}$ with $\varphi_{o}(1)=p$ and let $\rho_{o}$ be the associated Lempert projection. By [7, Proposition 3.4] it follows that

$$
\lim _{\mathbb{R} \ni r \rightarrow 1} k_{D}\left(\varphi(r), \rho_{o}(\varphi(r))\right)=0,
$$

namely, in the terminology of [1], the curve $\mathbb{R} \ni r \rightarrow \varphi(r)$ is special with respect to the projection $\rho_{o}$. In the proof of [7, Proposition 3.4] it is actually shown that the curve $r \mapsto \varphi(r)$ belongs eventually to any ball $\mathbb{B}$ contained in $D$ and tangent to $\partial D$ at $p$ and that

$$
\lim _{\mathbb{R} \ni r \rightarrow 1} \frac{\left\|\varphi(r)-\rho_{o}(\varphi(r))\right\|^{2}}{\operatorname{dist}\left(\rho_{o}(\varphi(r)), \partial D\right)}=0 .
$$

This condition, together with the fact that $r \mapsto \rho_{o}(\varphi(r))$ tends to $p$ non-tangentially in $D$ (and then in $\mathbb{B}$ ), guarantees that $\lim _{\mathbb{R} \ni r \rightarrow 1} k_{\mathbb{B}}\left(\varphi(r), \rho_{o}(\varphi(r))\right)=0$ (see [4] 
and [1, Proposition 2.7.10]). Since $E_{D}$ is strongly convex at $p$ (see Remark 4.2), there exists $\mathbb{B} \subset E_{D}$ tangent to $\partial D$ at $p$. Therefore

$$
\lim _{\mathbb{R} \ni r \rightarrow 1} k_{E_{D}}\left(\varphi(r), \rho_{o}(\varphi(r))\right) \leq \lim _{\mathbb{R} \ni r \rightarrow 1} k_{\mathbb{B}}\left(\varphi(r), \rho_{o}(\varphi(r))\right)=0 .
$$

Now, $\varphi \circ \theta_{R_{1}}: \mathbb{D} \rightarrow D$ is a complex geodesic in $E_{D}$ (here $\theta_{R_{1}}$ is defined in (3) and $R_{1}=\operatorname{Rr}(\varphi(0))$ ) and by (8) the map $\rho_{o}: E_{D} \rightarrow E_{D}$ is a holomorphic retraction, then

$$
\begin{aligned}
& \lim _{\mathbb{R} \ni r \rightarrow 1} k_{E_{D}}\left(\varphi(r), \rho_{o}(\varphi(r))\right) \\
& \left.\quad=\lim _{\mathbb{R} \ni r \rightarrow 1} k_{E_{D}}\left(\varphi \circ \theta_{R_{1}} \circ \theta_{R_{1}}^{-1}(r), \rho_{o} \circ \varphi \circ \theta_{R_{1}} \circ \theta_{R_{1}}^{-1}(r)\right)\right)=0 .
\end{aligned}
$$

Therefore we can write

$$
\begin{aligned}
k_{D}\left(z_{0}, \varphi(r)\right)-k_{E_{D}}\left(z_{0}, \varphi(r)\right)= & {\left[k_{D}\left(z_{0}, \rho_{o} \circ \varphi(r)\right)-k_{E_{D}}\left(z_{0}, \rho_{o} \circ \varphi(r)\right)\right] } \\
& +\left[k_{D}\left(z_{0}, \varphi(r)\right)-k_{D}\left(z_{0}, \rho_{o} \circ \varphi(r)\right)\right] \\
& -\left[k_{E_{D}}\left(z_{0}, \varphi(r)\right)-k_{E_{D}}\left(z_{0}, \rho_{o} \circ \varphi(r)\right)\right] .
\end{aligned}
$$

Since by (12),

$$
\lim _{r \rightarrow 1}\left|k_{D}\left(z_{0}, \varphi(r)\right)-k_{D}\left(z_{0}, \rho_{o} \circ \varphi(r)\right)\right| \leq \lim _{r \rightarrow 1} k_{D}\left(\varphi(r), \rho_{o}(\varphi(r))=0,\right.
$$

and similarly for $k_{E_{D}}\left(z_{0}, \varphi(r)\right)-k_{E_{D}}\left(z_{0}, \rho_{o} \circ \varphi(r)\right)$, then

$$
\begin{aligned}
& \lim _{\mathbb{R} \ni r \rightarrow 1^{-}}\left[k_{D}\left(z_{0}, \varphi(r)\right)-k_{E_{D}}\left(z_{0}, \varphi(r)\right)\right] \\
& \quad=\lim _{\mathbb{R} \ni r \rightarrow 1^{-}}\left[k_{D}\left(z_{0}, \rho_{o} \circ \varphi(r)\right)-k_{E_{D}}\left(z_{0}, \rho_{o} \circ \varphi(r)\right)\right] .
\end{aligned}
$$

Let $\tau(\zeta):=\varphi_{o}^{-1} \circ \rho_{o} \circ \varphi(\zeta)$. Then $\tau: \mathbb{D} \rightarrow \mathbb{D}$ is holomorphic, smooth at 1 and $\tau(1)=1$. Hence $0<\left|\tau^{\prime}(1)\right|<\infty$ and the classical Julia-Wolff-Carathéodory Theorem 2.2 implies

$$
\lim _{\mathbb{R} \ni r \rightarrow 1^{-}} \frac{|1-\tau(r)|}{1-|\tau(r)|}=\lim _{\mathbb{R} \ni r \rightarrow 1^{-}} \frac{|1-\tau(r)|}{1-r} \cdot \frac{1-r}{1-|\tau(r)|}=\left|\tau^{\prime}(1)\right| \cdot \frac{1}{\left|\tau^{\prime}(1)\right|}=1 .
$$

Therefore the curve $\mathbb{R} \ni r \mapsto \tau(r)$ converges radially to 1 .

For $r \in(0,1)$,

$$
\begin{aligned}
& k_{D}\left(z_{0}, \rho_{o} \circ \varphi(r)\right)-k_{E_{D}}\left(z_{0}, \rho_{o} \circ \varphi(r)\right) \\
& \quad=k_{D}\left(\varphi_{o}(0), \varphi_{o}(\tau(r))\right)-k_{E_{D}}\left(\varphi_{o} \circ \theta_{R} \circ \theta_{R}^{-1}(0), \varphi_{o} \circ \theta_{R} \circ \theta_{R}^{-1}(\tau(r))\right) \\
& \quad=\omega(0, \tau(r))-\omega\left(\theta_{R}^{-1}(0), \theta_{R}^{-1}(\tau(r))\right) .
\end{aligned}
$$

Using the explicit form of $\omega$, it follows that

$$
\lim _{\mathbb{D} \ni \zeta \rightarrow 1}\left[\omega(0, \zeta)-\omega\left(\theta_{R}^{-1}(0), \theta_{R}^{-1}(\zeta)\right)\right]=\lim _{\zeta \rightarrow 1} \frac{1}{2} \log \frac{1-\left|\Phi \circ \theta_{R}^{-1}(\zeta)\right|}{1-|\zeta|},
$$


where $\Phi(\zeta)=\left(\zeta-\theta_{R}^{-1}(0)\right) /\left(1-\theta_{R}^{-1}(0) \zeta\right)$. Now $\Phi \circ \theta_{R}^{-1}: E_{\mathbb{D}}(1,0, R) \rightarrow \mathbb{D}$ is holomorphic and $\Phi \circ \theta_{R}^{-1}(1)=1$. A direct calculation shows that

$$
\begin{aligned}
\lim _{\mathbb{R} \ni r \rightarrow 1^{-}} \frac{1-\left|\Phi \circ \theta_{R}^{-1}(r)\right|}{1-r} & =\lim _{\mathbb{R} \ni r \rightarrow 1^{-}} \frac{1-\Phi \circ \theta_{R}^{-1}(r)}{1-r} \\
& =\left.\left(\Phi \circ \theta_{R}^{-1}(\zeta)\right)^{\prime}\right|_{\zeta=1}=\frac{R-1}{R} .
\end{aligned}
$$

Since $\mathbb{R} \ni r \mapsto \tau(r)$ converges radially to 1 , by Lemma 2.4 it follows that

$$
\lim _{\mathbb{R} \ni r \rightarrow 1^{-}} \frac{1-\left|\Phi \circ \theta_{R}^{-1}(\tau(r))\right|}{1-|\tau(r)|}=\frac{R-1}{R},
$$

and from (14) we get the assertion.

\section{Mapping horospheres onto horospheres}

Let $D, D^{\prime}$ be two strongly convex bounded domains in $\mathbb{C}^{N}$ with smooth boundary. Let $p \in \partial D$ and $q \in \partial D^{\prime}$. We are going to study biholomorphisms between $E_{D}\left(p, z_{0}, R_{0}\right)$ and $E_{D^{\prime}}\left(q, z_{0}^{\prime}, R_{0}^{\prime}\right)$ such that $p$ is mapped (in the non-tangential sense) to $q$. First of all we give the following

Example 5.1. Let $\theta_{R}: \mathbb{D} \rightarrow E_{\mathbb{D}}(1,0, R) \subset \mathbb{D}$ be defined by (3). Let $R^{\prime}>0$. Then $\theta_{R}$ is a biholomorphism between $E_{\mathbb{D}}\left(1,0, R^{\prime}\right)$ and $\theta_{R}\left(E_{\mathbb{D}}\left(1,0, R^{\prime}\right)\right)$ which is a horosphere of $\mathbb{D}$. Moreover $\theta_{R}(1)=1$. However clearly $\theta_{R}$ does not extend to a biholomorphism from $\mathbb{D}$ to $\mathbb{D}$.

The problem with the previous example is that the corresponding horospheres have "different radii" as we explain in the following remark.

Remark 5.2. Let $F: D \rightarrow D^{\prime}$ be a biholomorphism between bounded strongly convex domains with smooth boundary. Let $p \in \partial D$. Then $F$ has non-tangential limit $q$ at $p$ for some $q \in \partial D^{\prime}$. This is pretty well known, actually $F$ extends smoothly on $D$ near $p$ (see [11] or [15, section 10]). Let $E_{D}$ be a horosphere of center $p$ and radius $R>0$ with respect to some pole $z_{1} \in E_{D}$. Then $F\left(E_{D}\right)$ is the horosphere $E_{D^{\prime}}$ of center $q$ and radius $R$ with respect to the pole $F\left(z_{1}\right)$. Indeed for any $z \in D$ we have

$$
\begin{aligned}
\lim _{w \rightarrow p}\left[k_{D}(z, w)-k_{D}\left(z_{1}, w\right)\right] & =\lim _{w \rightarrow p}\left[k_{D^{\prime}}(F(z), F(w))-k_{D^{\prime}}\left(F\left(z_{1}\right), F(w)\right)\right] \\
& =\lim _{w^{\prime} \rightarrow q}\left[k_{D^{\prime}}\left(F(z), w^{\prime}\right)-k_{D^{\prime}}\left(F\left(z_{1}\right), w^{\prime}\right)\right] .
\end{aligned}
$$

Let $E_{D}:=E_{D}\left(p, z_{0}, R_{0}\right)$ and $E_{D^{\prime}}:=E_{D^{\prime}}\left(q, z_{0}^{\prime}, R_{0}^{\prime}\right)$ be two horospheres of $D, D^{\prime}$ respectively. Assume that $z_{0} \in E_{D}$ and $z_{0}^{\prime} \in E_{D^{\prime}}$. For $z \in E_{D}$ denote by $R(z)$ the radius of $E_{D}$ with pole $z$, namely:

$$
E_{D}\left(p, z_{0}, R_{0}\right)=E_{D}(p, z, R(z)),
$$


and similarly for $z^{\prime} \in E_{D^{\prime}}$. Let $F: E_{D} \rightarrow E_{D^{\prime}}$ be a biholomorphism such that $F(p)=q$ (in the sense of non-tangential limits) and $F\left(z_{0}\right)=z_{0}^{\prime}$. As we have seen, the radius of a horosphere depends on the choice of its pole. It is not clear a priori that if $R_{0}=R_{0}^{\prime}$ then $R(z)=R(F(z))$ for all $z \in E_{D}$, unless $F$ is already known to be a biholomorphism between $D$ and $D^{\prime}$. This is however true, as we are going to show.

Lemma 5.3. Let $D, D^{\prime}$ be two strongly convex domains with smooth boundary, $p \in \partial D$ and $q \in \partial D^{\prime}$. Let $E_{D}:=E_{D}\left(p, z_{0}, R_{0}\right)$ and $E_{D^{\prime}}:=E_{D^{\prime}}\left(q, z_{0}^{\prime}, R_{0}^{\prime}\right)$ be two horospheres of $D, D^{\prime}$ respectively. Let $F: E_{D} \rightarrow E_{D^{\prime}}$ be a biholomorphism. If $R_{0}=R_{0}^{\prime}$ then for all $z \in E_{D}$ it follows $R(z)=R(F(z))$.

Proof. In (11) we saw that $R_{0}=R(z) / r(z)$ for all $z \in D$, where $r(z)$ is defined in (10). Similarly $R_{0}^{\prime}=R(F(z)) / r(F(z))$. Since $R_{0}=R_{0}^{\prime}$ we have

$$
\frac{R(z)}{r(z)}=\frac{R(F(z))}{r(F(z))} \text { for all } z \in E_{D} .
$$

Now we compute $r(z)$. Let $\varphi: \mathbb{D} \rightarrow D$ be a complex geodesic such that $\varphi(0)=z$ and $\varphi(1)=p$. Then by Lemma 4.6

$$
\begin{aligned}
\frac{1}{2} \log r(z)= & \lim _{w \rightarrow p}\left[k_{D}\left(z_{0}, w\right)-k_{D}(z, w)\right]=\lim _{\mathbb{R} \ni r \rightarrow 1}\left[k_{D}\left(z_{0}, \varphi(r)\right)-k_{D}(z, \varphi(r))\right] \\
= & \lim _{\mathbb{R} \ni r \rightarrow 1}\left[k_{D}\left(z_{0}, \varphi(r)\right)-k_{E_{D}}\left(z_{0}, \varphi(r)\right)\right] \\
& +\lim _{\mathbb{R} \ni r \rightarrow 1}\left[k_{E_{D}}\left(z_{0}, \varphi(r)\right)-k_{E_{D}}(z, \varphi(r))\right] \\
& -\lim _{\mathbb{R} \ni r \rightarrow 1}\left[k_{D}(z, \varphi(r))-k_{E_{D}}(z, \varphi(r))\right] \\
= & \frac{1}{2} \log \frac{R_{0}-1}{R_{0}}+\frac{1}{2} \log r_{E_{D}}(z) \\
& -\frac{1}{2} \log \frac{R(z)-1}{R(z)}=\frac{1}{2} \log \frac{R(z)\left(R_{0}-1\right)}{R_{0}(R(z)-1)} r_{E_{D}}(z) .
\end{aligned}
$$

Since $F$ is a biholomorphism, then it is an isometry between $k_{E_{D}}$ and $k_{E_{D}^{\prime}}$. Thus (taking $w \rightarrow p$ non-tangentially)

$$
\begin{aligned}
\frac{1}{2} \log r_{E_{D}}(z) & =\lim _{w \rightarrow p}\left[k_{E_{D}}\left(z_{0}, w\right)-k_{E_{D}}(z, w)\right] \\
& =\lim _{w \rightarrow p}\left[k_{E_{D}^{\prime}}\left(F\left(z_{0}\right), F(w)\right)-k_{E_{D}^{\prime}}(F(z), F(w))\right] \\
& =\frac{1}{2} \log r_{E_{D}^{\prime}}(F(z)) .
\end{aligned}
$$

Therefore, taking into account that $R_{0}=R_{0}^{\prime}$ we have

$$
\begin{aligned}
r(z) & =\frac{R(z)\left(R_{0}-1\right)}{R_{0}(R(z)-1)} r_{E_{D}}(z), \\
r(F(z)) & =\frac{R(F(z))\left(R_{0}-1\right)}{R_{0}(R(F(z))-1)} r_{E_{D}}(z)
\end{aligned}
$$


Substituting these into (15) we have $R(z)=R(F(z))$ as wanted.

Now we are in a good shape for stating and proving the following result:

Theorem 5.4. Let $D, D^{\prime}$ be bounded strongly convex domains in $\mathbb{C}^{N}$ with smooth boundary. Then $D$ is biholomorphic to $D^{\prime}$ if and only if there exist a horosphere $E_{D} \subset D$ with center $p \in \partial D$, a horosphere $E_{D^{\prime}} \subset D^{\prime}$ with center $q \in \partial D^{\prime}$ and a biholomorphism $F: E_{D} \rightarrow E_{D^{\prime}}$ such that $F(p)=q$ (in the sense of non-tangential limits) and the radius of $E_{D}$ with respect to some $z \in E_{D}$ is equal to the radius of $E_{D^{\prime}}$ with respect to $F(z)$.

Proof. One direction follows from Remark 5.2. As for the other direction, thanks to Lemma 3.3, we have just to show that there exists $\tilde{F}: D \rightarrow D^{\prime}$ smooth such that

(1) $\tilde{F}(z)=F(z)$ for all $z \in E_{D}$.

(2) $\zeta \mapsto \tilde{F}(\varphi(\zeta))$ is holomorphic for all $\varphi: \mathbb{D} \rightarrow D$ complex geodesics such that $\varphi(1)=p$.

We are going to define $\tilde{F}$ as follows. Let $\varphi_{v}: \mathbb{D} \rightarrow D$ be a complex geodesic as in section 3 (in particular given $z \in D$ there exist a unique $v \in \mathbb{C}^{N}$ and a unique $\zeta \in \mathbb{D}$ such that $\varphi_{v}(\zeta)=z$ ). Then $G_{E_{D}}=\varphi_{v}(\mathbb{D}) \cap E_{D}$ is a complex geodesic in $E_{D}$ by Lemma 4.4. Thus $F\left(G_{E_{D}}\right)$ is a complex geodesic in $E_{D^{\prime}}$. Let $t>0$ be such that $\varphi_{v}(t) \in E_{D}$. Let $\varphi_{F(v)}: \mathbb{D} \rightarrow D^{\prime}$ be the unique complex geodesic such that $\varphi_{F(v)}(1)=q$ and $\varphi_{F(v)}(t)=F\left(\varphi_{v}(t)\right)$. Again by Lemma 4.4, it is not too difficult to see that $\varphi_{F(v)}$ is independent of the point $t>0$ chosen to define it (with the property that $\varphi_{v}(t) \in E_{D}$ ). We let

$$
\tilde{F}\left(\varphi_{v}(\zeta)\right):=\varphi_{F(v)}(\zeta) .
$$

Notice that $\tilde{F}$ is well-defined by the uniqueness of the $\varphi_{v}$ 's. Also, by definition, property (2) follows, and, since the boundary Monge-Ampère foliations $\mathcal{F}_{p}, \mathcal{F}_{q}^{\prime}$ are smooth by Proposition 3.2 and $F$ is holomorphic, then $\tilde{F}$ is smooth.

We have only to show that $\tilde{F}(z)=F(z)$ for all $z \in E_{D}$. To see this, let $z \in E_{D}$ and let $\varphi_{v}: \mathbb{D} \rightarrow D$ be a complex geodesic such that $z \in \varphi_{v}(\mathbb{D})$. We can write $E_{D}=E_{D}\left(p, \varphi_{v}(0), r\right)$ for some $r>0$. By Lemma 5.3 then $E_{D^{\prime}}=E_{D^{\prime}}\left(q, F\left(\varphi_{v}(0)\right), r\right)$. Let $\theta_{r}: \mathbb{D} \rightarrow E_{\mathbb{D}}(1,0, r)$ be defined as in (3). By Remark 4.5 it follows that $\varphi_{v} \circ \theta_{r}: \mathbb{D} \rightarrow E_{D}$ is a complex geodesic of $E_{D}$ containing $z$ and then $F \circ \varphi_{v} \circ \theta_{r}: \mathbb{D} \rightarrow E_{D}^{\prime}$ is a complex geodesic of $E_{D^{\prime}}$ containing $F(z)$. Now, again by Remark 4.5 it follows that $\varphi_{F(v)} \circ \theta_{r}: \mathbb{D} \rightarrow E_{D^{\prime}}$ is a complex geodesic containing $F(z)$. We are then left to show that for all $\zeta \in \mathbb{D}$

$$
F \circ \varphi_{v} \circ \theta_{r}(\zeta)=\varphi_{F(v)} \circ \theta_{r}(\zeta) .
$$

But this is clear for they both map 1 to $q$ and $\theta_{r}^{-1}(0)$ to the same point $F\left(\varphi_{v}(0)\right)$. Thus (1) holds. 


\section{Plurisubharmonic solutions to the boundary Monge-Ampère equation}

The aim of this section is to study the solution of the complex Monge-Ampère equation $u: D \rightarrow \mathbb{R}$ such that $d u \neq 0,(\partial \bar{\partial} u)^{N}=0$ and $u$ is harmonic on each leaf of the boundary Monge-Ampère foliation at a given point $p \in \partial D$.

We begin with the following proposition, maybe interesting by its own.

Proposition 6.1. Let $D, D^{\prime} \subset \mathbb{C}^{N}$ be bounded strongly convex domains with smooth boundary, $p \in \partial D$ and $q \in \partial D^{\prime}$. Let $\Phi: D \rightarrow D^{\prime}$ be a smooth diffeomorphism, such that $\Phi$ has non-tangential limit $q$ at $p$. Suppose that $\Phi$ respects the boundary Monge-Ampère foliations of $D$ at $p$ and $D^{\prime}$ at $q$, namely

(1) $\Phi$ maps leaves of $\mathcal{F}_{p}$ onto leaves of $\mathcal{F}_{q}$ and it is a holomorphic Kobayashiisometry on each leaf,

(2) for any complex geodesic $\varphi: \mathbb{D} \rightarrow D$ such that $\varphi(1)=p$, it follows that

$$
\left\langle\varphi^{\prime}(1), n_{p}\right\rangle=\left\langle(\Phi \circ \varphi)^{\prime}(1), n_{q}^{\prime}\right\rangle,
$$

where $n_{p}\left(\right.$ respectively $n_{q}^{\prime}$ ) denotes the outer unit normal to $\partial D$ at $p$ (respectively to $\partial D^{\prime}$ at $q$ ).

Then $F$ maps horospheres of $D$ with center $p$ onto horospheres of $D^{\prime}$ with center $q$.

The second condition on $\Phi$ means that $\Phi$ cannot "squeeze" the complex geodesics. Note that by the first condition, $\Phi \circ \varphi$ extends smoothly on $\overline{\mathbb{D}}$ and thus it makes sense to consider $(\Phi \circ \varphi)^{\prime}(1)$.

Proof of Prop. 6.1. Let $E_{D}=E_{D}\left(p, z_{0}, R\left(z_{0}\right)\right)$ be a horosphere in $D$. We claim that

$$
\Phi\left(E_{D}\left(p, z_{0}, R\left(z_{0}\right)\right)\right)=E_{D^{\prime}}\left(q, \Phi\left(z_{0}\right), R\left(z_{0}\right)\right)=: E_{D^{\prime}} .
$$

Let $\varphi: \mathbb{D} \rightarrow D$ be a complex geodesic such that $\varphi(1)=p$. Let $z=\varphi(0)$. If we write $E_{D}=E_{D}(p, z, R(z))$ then $\varphi\left(E_{\mathbb{D}}(1,0, R(z))\right)=\varphi(\mathbb{D}) \cap E_{D}$. By hypothesis (1) $\Phi \circ \varphi: \mathbb{D} \rightarrow D^{\prime}$ is a complex geodesic in $D^{\prime}$. Thus $\Phi \circ \varphi$ extends smoothly past $\partial \mathbb{D}$ and since $\Phi$ has non-tangential limit $q$ at $p$ then $\Phi(\varphi(1))=q$.

Therefore, to show that $\Phi\left(E_{D}\right)=E_{D^{\prime}}$ is equivalent to proving that $\Phi\left(E_{D} \cap\right.$ $\varphi(\mathbb{D}))=E_{D^{\prime}} \cap \Phi(\varphi(\mathbb{D}))$ for any complex geodesic $\varphi: \mathbb{D} \rightarrow D$ such that $\varphi(1)=p$.

Thus we have to show that

$$
\Phi\left(\varphi\left(E_{\mathbb{D}}(1,0, R(z))\right)\right)=E_{D^{\prime}}(q, \Phi(z), R(\Phi(z))) \cap(\Phi \circ \varphi(\mathbb{D})) .
$$

In other words it is enough to show that $R(z)=R(\Phi(z))$ for all $z \in D$. By construction $R\left(z_{0}\right)=R\left(\Phi\left(z_{0}\right)\right)$ and by (11) we are left to show that $r(z)=r(\Phi(z))$ 
for all $z \in D$. Now, let $\varphi: \mathbb{D} \rightarrow D$ be a complex geodesic such that $\varphi(0)=z_{0}$ and $\varphi(1)=p$. Then

$$
\begin{aligned}
\frac{1}{2} \log r(z)= & \lim _{w \rightarrow p}\left[k_{D}\left(z_{0}, w\right)-k_{D}(z, w)\right]=\lim _{\mathbb{R} \ni r \rightarrow 1}\left[\omega(0, r)-k_{D}(z, \varphi(r))\right] \\
= & \lim _{\mathbb{R} \ni r \rightarrow 1}\left[k_{D^{\prime}}(\Phi \circ \varphi(0), \Phi \circ \varphi(r))-k_{D^{\prime}}(\Phi(z), \Phi \circ \varphi(r))\right] \\
& +\lim _{\mathbb{R} \ni r \rightarrow 1}\left[k_{D^{\prime}}(\Phi(z), \Phi \circ \varphi(r))-k_{D}(z, \varphi(r))\right] \\
= & \frac{1}{2} \log r(\Phi(z))+\lim _{\mathbb{R} \ni r \rightarrow 1}\left[k_{D^{\prime}}(\Phi(z), \Phi \circ \varphi(r))-k_{D}(z, \varphi(r))\right] .
\end{aligned}
$$

The assertion follows as soon as we prove that the limit in the last line of the previous formula is equal to 0 . To see this, let $\psi: \mathbb{D} \rightarrow D$ be the complex geodesic such that $\psi(1)=p$ and $\psi(0)=z$. Let $\rho: D \rightarrow D$ be the Lempert projection associated to $\psi$ and $\rho^{\prime}: D^{\prime} \rightarrow D^{\prime}$ be the Lempert projection associated to $\Phi \circ \psi$. By the triangle inequality and (12)

$$
\begin{aligned}
\lim _{r \rightarrow 1}[\mid & k_{D^{\prime}}(\Phi(z), \Phi \circ \varphi(r))-k_{D^{\prime}}\left(\Phi(z), \rho^{\prime} \circ \Phi \circ \varphi(r)\right) \mid \\
& \left.\quad+\left|k_{D}(z, \varphi(r))-k_{D}(z, \rho \circ \varphi(r))\right|\right] \\
\leq & \lim _{r \rightarrow 1}\left[k_{D^{\prime}}\left(\Phi \circ \varphi(r), \rho^{\prime} \circ \Phi \circ \varphi(r)\right)+k_{D}(\varphi(r), \rho \circ \varphi(r))\right]=0 .
\end{aligned}
$$

Thus, from the very definition of $\omega$, it follows that

$$
\begin{aligned}
& \lim _{\mathbb{R} \ni r \rightarrow 1}\left[k_{D^{\prime}}(\Phi(z), \Phi \circ \varphi(r))-k_{D}(z, \varphi(r))\right] \\
& =\lim _{\mathbb{R} \ni r \rightarrow 1}\left[k_{D^{\prime}}\left(\Phi(z), \rho^{\prime} \circ \Phi \circ \varphi(r)\right)-k_{D}(z, \rho \circ \varphi(r))\right] \\
& =\lim _{\mathbb{R} \ni r \rightarrow 1}\left[\omega\left(0, \psi^{-1} \circ \Phi^{-1} \circ \rho^{\prime} \circ \Phi \circ \varphi(r)\right)-\omega\left(0, \psi^{-1} \circ \rho \circ \varphi(r)\right)\right] \\
& =\lim _{\mathbb{R} \ni r \rightarrow 1} \frac{1}{2} \log \frac{1-\left|\psi^{-1} \circ \rho \circ \varphi(r)\right|}{1-\left|\psi^{-1} \circ \Phi^{-1} \circ \rho^{\prime} \circ \Phi \circ \varphi(r)\right|} .
\end{aligned}
$$

By the classical Julia-Wolff-Carathèodory Theorem 2.2 and [1, Lemma 2.6.44] (or see [4]) it follows that

$$
\begin{aligned}
\lim _{\mathbb{R} \ni r \rightarrow 1} & \frac{1}{2} \log \frac{1-\left|\psi^{-1} \circ \rho \circ \varphi(r)\right|}{1-\left|\psi^{-1} \circ \Phi^{-1} \circ \rho^{\prime} \circ \Phi \circ \varphi(r)\right|} \\
= & \lim _{\mathbb{R} \ni r \rightarrow 1} \frac{1}{2} \log \frac{1-\left|\psi^{-1} \circ \rho \circ \varphi(r)\right|}{1-r} \\
& -\lim _{\mathbb{R} \ni r \rightarrow 1} \frac{1}{2} \log \frac{1-\left|\psi^{-1} \circ \Phi^{-1} \circ \rho^{\prime} \circ \Phi \circ \varphi(r)\right|}{1-r} \\
= & \frac{1}{2} \log \frac{\left\langle\varphi^{\prime}(1), n_{p}\right\rangle}{\left\langle\psi^{\prime}(1), n_{p}\right\rangle} \cdot \frac{\left\langle(\Phi \circ \psi)^{\prime}(1), n_{q}^{\prime}\right\rangle}{\left\langle(\Phi \circ \varphi)^{\prime}(1), n_{q}^{\prime}\right\rangle},
\end{aligned}
$$

which is 0 by hypothesis (2). 
By Remark 3.4 we have the following result:

Corollary 6.2. The boundary spherical representation $\Phi: D \rightarrow \mathbb{B}^{N}$ maps horospheres of $D$ onto horospheres of $\mathbb{B}^{N}$.

Starting from this result we can solve the Monge-Ampère equation at the boundary.

Theorem 6.3. Let $D$ be a bounded strongly convex domain in $\mathbb{C}^{N}$ with smooth boundary and let $p \in \partial D$. The Monge-Ampère equation

$$
\left\{\begin{array}{l}
u \text { plurisubharmonic in } D \\
(\partial \bar{\partial} u)^{N} \equiv 0 \\
d u \neq 0 \\
u(z)=0 \text { for } z \in \partial D \backslash\{p\} \\
u(z) \approx\|p-z\|^{-1} \text { as } z \rightarrow p \text { non-tangentially }
\end{array}\right.
$$

has a solution $u \in C^{\infty}(D)$ such that $u(z)<0$ for all $z \in D$ and $(\partial \bar{\partial} u)^{N-1} \neq 0$. Moreover the level sets of $u$ are boundaries of the horospheres of $D$ with center $p$.

Proof. First assume $D=\mathbb{B}^{N}$ and $p=e_{1}$. Then we consider

$$
u_{0}(z):=-\frac{1-\|z\|^{2}}{\left|1-z_{1}\right|^{2}} \text {. }
$$

A straightforward calculation shows that $u_{0}$ is actually a solution of the boundary Monge-Ampère equation (17). Moreover by [1, Proposition 2.2.20] (see also [2]) it follows that $E_{\mathbb{B}^{N}}\left(e_{1}, 0, R\right)=\left\{u_{0}(z)<-1 / R\right\}$ and thus the level sets of $u_{0}$ are exactly the boundary of the horospheres centered at $e_{1}$. These horospheres are strongly convex. Let $P(\zeta)=\left(1-|\zeta|^{2}\right) /\left(|1-\zeta|^{2}\right)$ be the Poisson kernel in $\mathbb{D}$. Then the set $\{P(\zeta)>1 / R\}$ is the horosphere of center 1 and radius $R>0$ of $\mathbb{D}$. A simple calculation shows

$$
u_{0} \circ \eta_{v}(\zeta)=-\frac{1}{v_{1}^{2}} P(\zeta)
$$

where $v \in L_{e_{1}}$ and $\eta_{v}$ is defined in the third section. Thus $u_{0}$ is harmonic on the leaves of the boundary Monge-Ampère foliation $\mathcal{G}_{e_{1}}$. Now, for a strongly convex domain $D$, define

$$
u:=u_{0} \circ \Phi
$$

where $\Phi: D \rightarrow \mathbb{B}^{N}$ is the boundary spherical representation of Chang, Hu and Lee. Then $u \in C^{\infty}(D), d u \neq 0$ and $u(z)=0$ for $z \in \partial D \backslash\{p\}$. Since $\Phi$ is holomorphic on the leaves of the boundary Monge-Ampère foliation then $u$ is 
harmonic on such leaves. Moreover, by Corollary 6.2 it follows that the level sets of $u$ are the boundary of the horospheres centered at $p$.

Let $\varphi: \mathbb{D} \rightarrow D$ be a complex geodesic such that $\varphi(1)=p$. In [17, Proposition 11] Lempert constructs a biholomorphism $G$ from $D$ to a domain $G(D)$ which extends smoothly through $\partial D$ and such that $G \circ \varphi(\zeta)=(\zeta, 0, \ldots, 0)$ and the associated Lempert projection is $\tilde{\rho}\left(z_{1}, \ldots, z_{n}\right)=\left(z_{1}, 0, \ldots, 0\right)$ (note that the Lempert projection $\rho: D \rightarrow D$ associated to $\varphi$ is equal to $\left.G \circ \tilde{\rho} \circ G^{-1}\right)$. For short we call such coordinates the "Lempert coordinates". The domain $G(D)$ is no longer convex in general, but it is still convex near $G(\varphi(\partial \mathbb{D}))$. We claim that if $E_{D}=u^{-1}(-1 / R)$ is a horosphere with center $p$ then $G\left(\partial E_{D}\right)$ is convex near $G\left(\partial E_{D}\right) \cap G(\varphi(\overline{\mathbb{D}}))$. Indeed, let $w_{0} \in G\left(\partial E_{D}\right) \cap G(\varphi(\mathbb{D}))$ and let $H_{w_{0}}^{\mathbb{R}} \subset \mathbb{C} e_{1}$ be the real one-dimensional tangent space to $G\left(\partial E_{D}\right) \cap G(\varphi(\mathbb{D}))$ at $w_{0}$. Since $\tilde{\rho}\left(G\left(E_{D}\right)\right) \subset G\left(E_{D}\right) \cap G(\varphi(\mathbb{D}))$ and $\tilde{\rho}$ is linear then the real hyperplane given by $\left\{w \in \mathbb{C}^{n}: \tilde{\rho}(w)-w_{0} \in H_{w_{0}}^{\mathbb{R}}\right\}$ separates $w_{0}$ from $G\left(E_{D}\right)$, which is thus convex at $w_{0}$. Moreover this clearly implies that the complex tangent space

$$
T_{G(\varphi(\zeta))}^{\mathbb{C}}\left(\partial G\left(E_{D}(G(p), G(\varphi(\zeta)), 1)\right)=\operatorname{span}\left(\frac{\partial}{\partial z_{j}}\right)_{j=2, \ldots, N}\right.
$$

for any $\zeta \in \mathbb{D}$ (notice that $\varphi(\zeta) \in \partial E_{D}(p, \varphi(\zeta), 1)$ always).

We are going to show that $u$ is a plurisubharmonic solution of the MongeAmpère equation using the Lempert coordinates. Let $z=\left(z_{1}, 0, \ldots, 0\right) \in G(D)$. Since $\partial G\left(E_{D}\right)$ is (pseudo)convex, the matrix

$$
\left(\frac{\partial^{2} u}{\partial z_{j} \partial \bar{z}_{k}}(z)\right)_{j, k=2, \ldots, N} \text { is non-negative definite. }
$$

Since $u$ is harmonic on $\zeta \mapsto(\zeta, 0, \ldots, 0)$ then $\frac{\partial^{2} u}{\partial z_{1} \partial \bar{z}_{1}}(z)=0$. We are left to show that $\frac{\partial^{2} u}{\partial z_{k} \partial \bar{z}_{1}}(z)=0$ for $k=2, \ldots, N$. But this is obvious since, by (18), we have $d u\left(\frac{\partial}{\partial z_{k}}\right) \equiv 0$ on $(\zeta, 0, \ldots, 0), \zeta \in \mathbb{D}, k=2, \ldots, N$.

Therefore $u$ is plurisubharmonic in $D$, or, in other terms, $d d^{c} u=2 i \partial \bar{\partial} u \geq 0$. To show that $(\partial \bar{\partial} u)^{N-1} \neq 0$ it is enough to see that $d d^{c} u>0$ in $D$ on the complex tangent spaces to the boundary of horospheres in $D$. To this aim, we introduce the following functions: $\tau: D \rightarrow \mathbb{R}^{+}$and $\tau_{0}: \mathbb{B}^{N} \rightarrow \mathbb{R}^{+}$defined by $\tau(z)=\exp (u(z))$ and $\tau_{0}(z)=\exp \left(u_{0}(z)\right)$. A direct computation shows that $d d^{c} \tau_{0}>0$ in $\mathbb{B}^{N}$ and $d d^{c} \tau \geq 0$ in $D$. The form $d d^{c} u$ is then positive definite on the complex tangent spaces to the boundary of horospheres if and only if $\tau$ is strictly plurisubharmonic, namely, $d d^{c} \tau>0$ in $D$. Now, we claim that $\Phi$ is a "contact map", that is, it maps the complex tangent space of a (boundary of) horosphere of $\mathbb{B}^{N}$ to the complex tangent space of the corresponding (boundary of) horosphere. Indeed, up to composition with automorphisms of $\mathbb{B}^{N}$ we can assume that the complex geodesic $\eta(\zeta)=(\zeta, 0, \ldots, 0)$ of $\mathbb{B}^{N}$ is mapped to the 
complex geodesic $\varphi=\Phi^{-1} \circ \eta$ which, in Lempert's coordinates, is given by $\zeta \mapsto(\zeta, 0, \ldots, 0)$. With this choice of coordinates-and since biholomorphisms preserve complex tangent spaces-equation (18) implies that $\Phi$ is a contact map. In other words, $\left(\Phi^{-1}\right)^{*}(\partial \tau)=g \partial \tau_{0}$ for some smooth function $g$. To compute $g$, we consider an arbitrary complex geodesic $\eta: \mathbb{D} \rightarrow \mathbb{B}^{N}$ containing $e_{1}$. By definition, $\tau_{0} \circ \eta=\tau \circ \Phi^{-1} \circ \eta$. Therefore $\eta^{*}\left(\partial \tau_{0}\right)=\eta^{*}\left(\left(\Phi^{-1}\right)^{*} \partial \tau\right)=g \eta^{*}\left(\partial \tau_{0}\right)$ and thus $g \equiv 1$. Now, taking into account that $\bar{\partial} \partial=d \partial$, it follows that

$$
\begin{aligned}
d d^{c} \tau_{0} & =-2 i \bar{\partial} \partial \tau_{0}=-2 i d\left(\left(\Phi^{-1}\right)^{*}(\partial \tau)\right) \\
& =-2 i\left(\Phi^{-1}\right)^{*}(d \partial \tau)=\left(\Phi^{-1}\right)^{*}\left(d d^{c} \tau\right) .
\end{aligned}
$$

Hence $d d^{c} \tau$ is non-degenerate. Since we already know that $d d^{c} \tau \geq 0$, the only possibility is that $d d^{c} \tau>0$, as wanted.

Finally we show that $u(z) \approx\|p-z\|^{-1}$ as $z \rightarrow p$ non-tangentially. Let $\left\{z_{k}\right\} \subset D$ be a sequence converging to $p$ non-tangentially. We use the notations $\varphi_{v}, \eta_{v}$ for $v \in L_{p}$ as introduced in Section 3. Let $\zeta_{k} \in \mathbb{D}$ and $v_{k} \in L_{p}$ be such that $\varphi_{v_{k}}\left(\zeta_{k}\right)=z_{k}$. Since the leaves of $\mathcal{F}_{p}$ are transversal to $\partial D$ at $p$ and $z_{k} \rightarrow p$ non-tangentially, up to subsequences, we may assume that $v_{k} \rightarrow v_{0} \in L_{p}$. Also, $\zeta_{k} \rightarrow 1$ non-tangentially (since complex geodesics maps non-tangential regions onto non-tangential regions, see, e.g., [1, Proposition 2.7.8.(ii)]). Thus $\eta_{v_{k}} \rightarrow \eta_{v_{0}}$ as $v_{k} \rightarrow v_{0}$ and $\Phi\left(z_{k}\right) \rightarrow e_{1}$ non-tangentially. Write

$$
u(z) \cdot\left\|p-z_{k}\right\|=u_{0}\left(\Phi\left(z_{k}\right)\right)\left\|e_{1}-\Phi\left(z_{k}\right)\right\| \cdot \frac{\left\|p-z_{k}\right\|}{\left\|e_{1}-\Phi\left(z_{k}\right)\right\|} .
$$

Then

$$
u_{0}\left(\Phi\left(z_{k}\right)\right) \cdot\left\|e_{1}-\Phi\left(z_{k}\right)\right\|=\frac{1-\left\|\Phi\left(z_{k}\right)\right\|^{2}}{\left|1-\Phi_{1}\left(z_{k}\right)\right|} \cdot \frac{\left\|e_{1}-\Phi\left(z_{k}\right)\right\|}{\left|1-\Phi_{1}\left(z_{k}\right)\right|}
$$

and both these factors are bounded away from zero and infinity since $\Phi\left(z_{k}\right) \rightarrow e_{1}$ non-tangentially (see, e.g., [1, Section 2.2.3]).

Now we examine the term $\frac{\left\|p-z_{k}\right\|}{\left\|e_{1}-\Phi\left(z_{k}\right)\right\|}$. First of all, since $z_{k} \rightarrow p, \Phi\left(z_{k}\right) \rightarrow e_{1}$ non-tangentially then $\left\|p-z_{k}\right\| \approx d\left(z_{k}, \partial D\right)$ and $\left\|e_{1}-\Phi\left(z_{k}\right)\right\| \approx d\left(\Phi\left(z_{k}\right), \partial \mathbb{B}^{N}\right)$, where $d(\cdot, \partial D)$ is the (euclidean) distance. By the boundary localization estimates for the Kobayashi distance (see, e.g. [1]) we know that $-\log d\left(z_{k}, \partial D\right) \approx$ $k_{D}\left(z_{0}, z_{k}\right)$ for any $z_{0} \in D$ fixed. Therefore, passing to the logarithm, we are left to show that there exist $c, C>0$ such that for all $k \in \mathbb{N}$

$$
-c<k_{D}\left(z_{0}, z_{k}\right)-k_{\mathbb{B}^{N}}\left(0, \Phi\left(z_{k}\right)\right)<C .
$$

Now, $z_{k}=\varphi_{v_{k}}\left(\zeta_{k}\right)$. Since $\varphi_{v_{k}}(0) \rightarrow \varphi_{v_{0}}(0)$ then the triangle inequality implies

$$
\begin{aligned}
& \left|k_{D}\left(\varphi_{v_{k}}\left(\zeta_{k}\right), z_{0}\right)-\omega\left(0, \zeta_{k}\right)\right| \\
& \quad=\left|k_{D}\left(\varphi_{v_{k}}\left(\zeta_{k}\right), z_{0}\right)-k_{D}\left(\varphi_{v_{k}}\left(\zeta_{k}\right), \varphi_{v_{k}}(0)\right)\right|<\mathrm{const}<\infty .
\end{aligned}
$$

Similarly, since $\Phi\left(z_{k}\right)=\eta_{v_{k}}\left(\zeta_{k}\right)$ we have that $k_{\mathbb{B}^{N}}\left(0, \Phi\left(z_{k}\right)\right) \approx \omega\left(0, \zeta_{k}\right)$ and thus (19) follows. 
Remark 6.4. From the proof of Theorem 6.3 it follows that the boundaries of horospheres are smooth strongly pseudoconvex, actually strongly convex for big radii.

\section{Some applications}

The first application is somehow a rephrasing of Theorem 6.3 in terms of the Busemann functions of $D$. We thank Stefano Trapani for explaining this point to us.

Proposition 7.1. Let $D \subset \mathbb{C}^{N}$ be a bounded strongly convex domain with smooth boundary. Let $u$ be the solution of (17) in $D$ given by Theorem 6.3. Let $\varphi: \mathbb{D} \rightarrow D$ be a complex geodesic such that $\varphi(1)=p \in \partial D$. Let $B_{\varphi}$ be the Busemann function of $\varphi$ at $p$. Then for all $z \in D$ it follows that

$$
B_{\varphi}(z)=-\frac{1}{2}(\log |u(z)|-\log |u(\varphi(0))|) .
$$

Proof. By the very definition, the level sets of $B_{\varphi}$ and $u$ are the same. Therefore there exists $\beta: \mathbb{R} \rightarrow \mathbb{R}$ such that $\log |u(z)|=\beta\left(B_{\varphi}(z)\right)$ for all $z \in D$. We are going to compute $\beta$. By the very definition of $B_{\varphi}$, we have $B_{\varphi}(\varphi(-t))=\omega(0, t)$ for all $t \in(0,1)$. Therefore $\beta(\omega(0, t))=\log |u(\varphi(-t))|$. Let $\varphi_{v}$ be the complex geodesic defined in Section 3 such that $\varphi=\varphi_{v} \circ \theta$ for some automorphism $\theta$ of $\mathbb{D}$ with $\theta(1)=1$. As shown in the proof of Theorem 6.3 it follows that $\left|u\left(\varphi_{v}(\theta(\zeta))\right)\right|=\frac{1}{v_{1}^{2}} P(\theta(\zeta))$ for all $\zeta \in \mathbb{D}$, where $P$ is the Poisson kernel in $\mathbb{D}$. By Lemma 2.1 it follows $P(\theta(\zeta))=\alpha P(\zeta)$ for some $\alpha>0$. In particular $|u(\varphi(0))|=P(0) \alpha / v_{1}^{2}=\alpha / v_{1}^{2}$. Therefore, taking into account that $\omega(0, t)=\frac{1}{2} \log \frac{1+t}{1-t}$, a direct computation shows that $\beta(s)=-2 s+\log \left(\alpha / v_{1}^{2}\right)$ and formula (20) is proved.

Remark 7.2. Formula (20) can be used to relate the work of Trapani (see [21]) with our solution $u$ of the boundary Monge-Ampère equation. The story goes as follows. For $z \in D$ let $\varphi_{z}: \mathbb{D} \rightarrow D$ be the unique complex geodesic such that $\varphi_{z}(1)=p$ and $\varphi_{z}(0)=z$. Let $v_{z}=\left.\frac{\partial \varphi_{z}\left(e^{i \theta}\right)}{\partial \theta}\right|_{\theta=0}$. Then $v_{z} \in T_{p} \partial D$. All the vectors $v_{z}$ belong to the same half-space $\mathcal{D}_{p}$ of $T_{p} \partial D$ with respect to the decomposition of this one induced by $T_{p}^{\mathbb{C}} \partial D$. Let $\Theta$ be a real linear form vanishing on $T_{p}^{\mathbb{C}} \partial D$ and positive on $\mathcal{D}_{p}$. Any other such a form is multiple of $\Theta$ by a positive constant. Define $\Psi: D \rightarrow \mathbb{R}$ as

$$
\Psi(z):=-\Theta\left(v_{z}\right)
$$

In [21, Theorem 4.1] it is proved that $B_{\varphi}(z)=\frac{1}{2}(\log |\Psi(z)|-\log |\Psi(0)|)$. Formula (20) implies then that $u(z)=C / \Psi(z)$ for some $C \in \mathbb{R}^{+} \backslash\{0\}$. 
As a second application we show that our solution of (17) can be used to characterize biholomorphisms, exactly as the Poisson kernel characterizes automorphism of the unit disc $\mathbb{D}$. Indeed, Lemma 2.1 can be rephrased as: $f: \mathbb{D} \rightarrow \mathbb{D}$ holomorphic, $f(1)=1$ (in the sense of non-tangential limits) is an automorphism of $\mathbb{D}$ if and only if there exists $\lambda \in \mathbb{R}^{+} \backslash\{0\}$ such that $f^{*}(P(\zeta))=\lambda P(\zeta)$ for all $\zeta \in \mathbb{D}$ (here $P$ is the Poisson kernel of $\mathbb{D}$ ).

Theorem 7.3. Let $D, D^{\prime} \subset \mathbb{C}^{N}$ be bounded strongly convex domains with smooth boundary. Let $p \in \partial D$ and $q \in \partial D^{\prime}$. Let $u_{D}$ (respectively $u_{D^{\prime}}$ ) be the solution of (17) in $D$ (respectively in $D^{\prime}$ ) given by Theorem 6.3. Let $F: D \rightarrow D^{\prime}$ be holomorphic and assume that $F$ is continuous at $p$. Then $F$ is a biholomorphism such that $F(p)=q$ if and only if there exists $\lambda \in \mathbb{R}^{+} \backslash\{0\}$ such that $F^{*}\left(u_{D^{\prime}}\right)=\lambda u_{D}$.

Proof. If $F$ is a biholomorphism such that $F(p)=q$ then it maps horospheres in $D$ with center $p$ onto horospheres in $D^{\prime}$ with center $q$. Let $\varphi: \mathbb{D} \rightarrow D$ be a complex geodesic and set $\varphi^{\prime}:=\varphi \circ F$, a complex geodesic for $D^{\prime}$. The biholomorphic invariance of the Kobayashi distance immediately yields $B_{\varphi}(z)=B_{\varphi^{\prime}}(F(z))$, where $B_{\varphi}$ is the Busemann function of $\varphi$ (and similarly $B_{\varphi^{\prime}}$ ). Proposition 7.1 then gives

$$
\left|F^{*}\left(u_{D^{\prime}}\right)\right|=\frac{\left|u_{D^{\prime}}(F(\varphi(0)))\right|}{|u(\varphi(0))|}\left|u_{D}\right|,
$$

and the assertion follows because both $u_{D}, u_{D^{\prime}}$ are strictly negative.

Conversely, first we show that the hypothesis $F^{*}\left(u_{D^{\prime}}\right)=\lambda u_{D}$ implies that $F(p)=q$. Indeed, let $\left\{z_{k}\right\} \subset D$ be a sequence converging non-tangentially to $p$. Up to extracting subsequences, we may assume that $\left\{F\left(z_{k}\right)\right\}$ is converging to $x \in \overline{D^{\prime}}$. Now

$$
\lambda u_{D}\left(z_{k}\right)=u_{D^{\prime}}\left(F\left(z_{k}\right)\right),
$$

therefore if $x \neq q$ it follows that the right-hand side of (21) stays bounded as $k \rightarrow \infty$ while the left-hand side diverges, which is impossible. Thus $F$ has nontangential limit $q$ at $p$. The hypothesis that $F$ is continuous at $p$ implies then that $F(p)=q$.

Secondly, the hypothesis $F^{*}\left(u_{D^{\prime}}\right)=\lambda u_{D}$ implies that for $z \in D, R>0$ there exists $a=a(z)>0$ such that

$$
F\left(\partial E_{D}(p, z, R)\right) \subseteq \partial E_{D^{\prime}}(q, F(z), a R) .
$$

Notice that, once $z$ is fixed, $a$ is independent of $R>0$. Indeed, for all $t<0$ it follows $F^{-1}\left(u_{D^{\prime}}^{-1}(t)\right)=u_{D}^{-1}(t / \lambda)$.

Now, let $\varphi: \mathbb{D} \rightarrow D$ be a complex geodesic such that $\varphi(1)=p$ and let $z_{0}=\varphi(0)$. Let $\psi: \mathbb{D} \rightarrow D^{\prime}$ be a complex geodesic such that $\psi(1)=q$ and $\psi(0)=F\left(z_{0}\right)$ and let $\rho: D^{\prime} \rightarrow \psi(\mathbb{D})$ be the associated Lempert projection. Consider the holomorphic function $f: \mathbb{D} \rightarrow \mathbb{D}$ defined as $f(\zeta)=\psi^{-1} \circ \rho \circ F \circ \varphi(\zeta)$. 
Then $f$ is continuous at 1 and clearly $f(1)=1$. We claim that $f$ is an automorphism of $\mathbb{D}$. To see this, thanks to Lemma 2.1 , it is enough to show that there exists $\alpha>0$ such that for all $R>0$,

$$
f\left(E_{\mathbb{D}}(1,0, R)\right) \subseteq E_{\mathbb{D}}(1,0, \alpha R),
$$

and that there exists a point $\zeta_{0} \in \partial E_{\mathbb{D}}(1,0, R)$ such that $f\left(\zeta_{0}\right) \in \partial E_{\mathbb{D}}(1,0, \alpha R)$ for some $R>0$.

Let $R>0$. By Remark 4.5, it follows $\varphi\left(\partial E_{\mathbb{D}}(1,0, R)\right)=\partial E_{D}\left(p, z_{0}, R\right) \cap$ $\varphi(\overline{\mathbb{D}})$. Thus by (22), setting $\alpha=a\left(z_{0}\right)$,

$$
F \circ \varphi\left(\partial E_{\mathbb{D}}(1,0, R) \backslash\{1\}\right) \subset \partial E_{D^{\prime}}\left(q, F\left(z_{0}\right), \alpha R\right) \cap F(\varphi(\overline{\mathbb{D}} \backslash\{p\})) .
$$

By (8) we have that $\rho\left(\partial E_{D^{\prime}}\left(q, F\left(z_{0}\right), \alpha R\right)\right) \subset \overline{E_{\mathbb{D}}(1,0, \alpha R)}$ and thus formula (23) holds. It is then clear by construction that $0 \in \partial E_{\mathbb{D}}(1,0,1)$ and $f(0) \in \partial E_{\mathbb{D}}(1,0, \alpha)$ and thus $f$ is an automorphism of $\mathbb{D}$.

Next aim is to show that $F \circ \varphi: \mathbb{D} \rightarrow D^{\prime}$ is a complex geodesic. For what we have just shown, it follows that $\rho \circ F \circ \varphi=\psi \circ f: \mathbb{D} \rightarrow D^{\prime}$ is a complex geodesic. Thus for all $\zeta_{1}, \zeta_{2} \in \mathbb{D}$

$$
\begin{aligned}
\omega\left(\zeta_{1}, \zeta_{2}\right) & =k_{D^{\prime}}\left(\rho\left(F\left(\varphi\left(\zeta_{1}\right)\right)\right), \rho\left(F\left(\varphi\left(\zeta_{2}\right)\right)\right)\right) \\
& \leq k_{D^{\prime}}\left(F\left(\varphi\left(\zeta_{1}\right)\right), F\left(\varphi\left(\zeta_{2}\right)\right)\right) \leq \omega\left(\zeta_{1}, \zeta_{2}\right),
\end{aligned}
$$

which implies $k_{D^{\prime}}\left(F\left(\varphi\left(\zeta_{1}\right)\right), F\left(\varphi\left(\zeta_{2}\right)\right)\right)=\omega\left(\zeta_{1}, \zeta_{2}\right)$ and thus $F \circ \varphi: \mathbb{D} \rightarrow D^{\prime}$ is a complex geodesic. Therefore $F$ maps complex geodesics onto complex geodesics.

To show that $F$ is injective we have only to show that if $\varphi, \eta: \mathbb{D} \rightarrow D$ are complex geodesics such that $\varphi(1)=\eta(1)=p$ and $\varphi(\mathbb{D}) \cap \eta(\mathbb{D})=\emptyset$ then $F(\varphi(\mathbb{D})) \cap$ $F(\eta(\mathbb{D}))=\emptyset$. Suppose this is not the case. Since $F \circ \varphi, F \circ \eta: \mathbb{D} \rightarrow D^{\prime}$ are complex geodesics, then $F(\varphi(\mathbb{D})) \cap F(\eta(\mathbb{D})) \neq \emptyset$ implies $F(\varphi(\mathbb{D}))=F(\eta(\mathbb{D}))$. Let $\rho_{a}: D^{\prime} \rightarrow D^{\prime}$ be the Lempert projection associated to $h:=F \circ \varphi$. Consider the holomorphic map $\tilde{\rho}: D \rightarrow \mathbb{D}$ defined as

$$
\tilde{\rho}(z):=h^{-1} \circ \rho_{a} \circ F(z) .
$$

It is clear that $\tilde{\rho} \circ \varphi(\zeta)=\zeta$ for all $\zeta \in \mathbb{D}$. Since $\tilde{\rho}(\eta(\mathbb{D}))=\mathbb{D}$ by construction, it follows $\varphi(\mathbb{D})=\eta(\mathbb{D})$, which is a contradiction.

It remains to show that $F$ is surjective. First, since $D$ and $D^{\prime}$ have the same dimension, it follows that $F(D)$ is open. Assume that $F$ is not surjective and let $w \notin F(D)$ be such that $w \in \partial F(D) \cap D^{\prime}$. Then there exists a sequence $\left\{z_{k}\right\} \subset D$ such that $F\left(z_{k}\right) \rightarrow w$. Up to subsequences assume that $z_{k} \rightarrow z_{0} \in \bar{D}$. It is clear that $z_{0} \in \partial D$ for otherwise $F\left(z_{0}\right)=w$. Also, since $F(p)=q$ then $z_{0} \neq p$. By hypothesis $\lambda u_{D}\left(z_{k}\right)=u_{D^{\prime}}\left(F\left(z_{k}\right)\right)$. But $u_{D}\left(z_{k}\right) \rightarrow u_{D}\left(z_{0}\right)=0$ while $u_{D^{\prime}}\left(F\left(z_{k}\right)\right) \rightarrow u_{D^{\prime}}(w)<0$ as $k \rightarrow \infty$, giving a contradiction. 
Acknowledgements. We thank very much Stefano Trapani for carefully reading a preliminary version of this manuscript and for many helpful comments which substantially improved the paper. Also, we are grateful to the referee for his/her precise suggestions.

\section{References}

1. Abate, M.: Iteration theory of holomorphic maps on taut manifolds. Mediterranean Press, Rende, Cosenza, 1989

2. Abate, M.: Horospheres and iterates of holomorphic maps. Math. Z. 198, 225-238 (1988)

3. Abate, M.: Common fixed points of commuting holomorphic maps. Math. Ann. 283, 645655 (1989)

4. Abate, M.: The Lindelöf principle and the angular derivative in strongly convex domains. J. Analyse Math. 54, 189-228 (1990)

5. Abate, M., Tauraso, R.: The Lindelöf principle and angular derivatives in convex domains of finite type. J. Austr. Math. Soc. 73, 221-250 (2002)

6. Bland, J., Duchamp, T., Kalka, M.: On the automorphism group of strictly convex domains in $\mathbb{C}^{n}$. Contemp. Math. 49, 19-30 (1986)

7. Bracci, F.: Commuting holomorphic maps in strongly convex domains. Ann. Scuola Norm. Sup. Pisa Cl. Sci. (4), XXVII, 1998, pp. 131-144

8. Bracci, F.: Dilatation and order of contact for holomorphic self-maps of strongly convex domains. Proc. London Math. Soc. 86(1), 131-152 (2003)

9. Busemann, H.: The Geometry of Geodesics. Academic Press - New York, 1955

10. Chang, C.H., Hu, M.C., Lee, H.P.: Extremal analytic discs with prescribed boundary data. Trans. Amer. Math. Soc. 310(1), 355-369 (1988)

11. Fefferman, C.: The Bergman kernel and biholomorphic mappings of pseudoconvex domains. Invent. Math. 26, 1-65 (1974)

12. Heins, M.H.: A theorem of Wolff-Denjoy type. Complex Analysis, Birkhäuser, Basel, 1988, pp. $81-86$

13. Herzig, A.: Die Winkelderivierte und das Poisson-Stieltjes Integral. Math. Z. 46, 129-156 (1940)

14. Huang, X.: A non-degeneracy property of extremal mappings and iterates of holomorphic self-mappings. Ann. Scuola Norm. Sup. Pisa Cl. Sci. (4) 21(3) 399-419 (1994)

15. Lempert, L.: La métrique de Kobayashi et la representation des domaines sur la boule. Bull. Soc. Math. Fr. 109, 427-474 (1981)

16. L. Lempert: Holomorphic retracts and intrinsic metrics in convex domains. Analysis Math. 8 (1982), 257-261.

17. L. Lempert: Intrinsic distances and holomorphic retracts. Complex Analysis and Applications'81, Sofia, (1984), 341-364.

18. Leung, K.-W., Patrizio, G., Wong, P.-M.: Isometries of intrinsic metrics on strictly convex domains. Math. Z. 196, 343-353 (1987)

19. Patrizio, G.: Parabolic exhaustion for strictly convex domains. Manuscr. Math. 47, 271-309 (1984)

20. Patrizio, G.: A characterization of complex manifolds biholomorphic to a circular domain. Math. Z. 189 343-363 (1985)

21. Trapani, S.: Dual maps and Kobayashi distance of bounded convex domains in $\mathbb{C}^{n}$. Proc. of the Workshop "Contemporary Geometry and Related Topics", Belgrado, Jugoslavia 2002. World Scientific 2004

22. Vigué, J.-P.: Sur les rétractes holomorphes de dimension 1. Rend. Mat. Acc. Lincei 9, 31-41 (1998) 